\title{
Is proximity preservation rational in social choice theory?
}

\author{
Wu-Hsiung Huang \\ Department of Mathematics, National Taiwan University, Taipei 106, Taiwan \\ (e-mail: whuang@math.htu.edu.tw)
}

Received: 17 February 1999/Accepted: 27 January 2003

\begin{abstract}
We establish a strong impossibility theorem of a rational social choice that the proximity preservation (also called weak proximorphism WPX) and the diagonal surjectivity are logically inconsistent. The result is valid for finite or infinite alternatives, discrete or continuous. It generalizes the Baigent theorem, largely weakening his antecedent. For continuum set of alternatives, we clarify the notion of WPX by showing (1) WPX almost implies the continuity, (2) WPX is almost rigid. These observations raise the issue whether WPX is a natural condition for a social welfare function. A splitting reformulation of the proximity preservation which is weaker but rational is suggested.
\end{abstract}

\section{Introduction}

Chichilnisky (1982) showed in a topological framework that there exits no social welfare function which is continuous, unanimous and anonymous. ${ }^{1}$ Analogous to it, Baigent (1987) introduced for the finite discrete case of alternatives a notion of proximity preservation to substitute the continuity condition and showed that the proximity preservation, the unanimity and anonymity are also logically inconsistent.

In this paper, we extend Baigent's result by proving (see Theorem 2) that the proximity preservation (WPX) and the diagonal surjectivity (DS) are inconsistent; this is an improvement because the hypothesis of anonymity is dropped and the diagonal surjectivity ${ }^{2}$ is considerably weaker than the

\footnotetext{
${ }^{1}$ For precise definitions, see [3] or Appendix I.

${ }^{2}$ The diagonal surjectivity means that the social welfare function maps unanimous profiles of individual preferences onto social preferences.
} 
unanimity. Even stronger, our result is valid also for the case of infinite alternatives, discrete or continuous.

The result (Theorem 2) in its appearance is a strong impossibility theorem of rational social choice. However, it also forces us to question whether the proximity preservation is a natural condition to be imposed on a social welfare function. Indeed, it states that a condition as weak as the diagonal surjectivity is enough to preclude the proximity preservation. The argument is purely geometric, involving essentially no concept of social choice theory. Consequently, could one say that a condition is natural, or even rational, when it hardly respects simple geometry?

From a version of structuralism, we consider the proximity structure first introduced by Baigent (1987) and define (see 1.1) a function proximorphic if it preserves the proximity structure in a canonical way. The proof of Theorem 1 provides a short and clear argument to show how a diagonally surjective function is not proximorphic. Baigent defined proximity preservation in a weaker sense; we call a function satisfying his condition weakly proximorphic in this paper. Theorem 2 deals with weak proximorphisms as an extension of Theorem 1 for $N \geq 3$, where $N$ is the number of the individuals in the society. Its proof is essentially the same, but technically more subtle and tedious than that of Theorem 1 .

Since for social choice theory, the number $N$ is usually assumed to be at least three, Theorem 3 , which treats $N=2$ is purely of geometric interest. It shows that a diagonally surjective weak proximorphism must be simple, i.e the elements of the preference space must be equally distanced from each other and the map is simply a permuted projection (see Definition 2.1).

The latter part (Sect. 3 and Sect. 4) of this paper is devoted toward clarifying the notion of proximity preservation on continuum spaces. As the proximity preservation and the continuity are designed to be analogous, we first compare their geometric behaviors on canonical spaces, such as on the $P$-spaces (see Definition 3.1) with countable bases, and in particular on the Euclidean spaces. It is proved (in Theorem 4 and Corollary 1) that the proximity preservation implies the continuity on the canonical spaces. In the framework of Chichilnisky where the range space is a $P$-space but has no countable base, the extreme case that the social welfare function (SWF) is totally discrete (or even scattering) may occur. Theorem 5 states that on each component of the Chichilnisky space, a SWF preserving proximity is either continuous or scattering. In particular, if a SWF is assumed to be onto (i.e., to have no taboo), then the proximity preservation implies continuity. The converse is clearly false, since the proximity preservation is almost rigid but the continuity allows a large extent of elasticity.

In Sect. 4, we show a series of theorems for completion. They examine more closely the geometry of weak proximorphisms. We introduce the notion of path-minimizing, and show that the proximity preservation yields almost what we call "similarity" in the elementary geometry. In particular, there is no function $F$ mapping a domain of the Euclidean space $\mathbb{R}^{n}$ into $\mathbb{R}^{m}, n>m$, which preseves proximity, unless $F$ is trivial. Again, this reveals that the 
proximity preservation is a strict condition even in a pure geometric viewpoint.

We add the last section for concluding remarks, where the recent works by Grafe and Grafe[5] and by Ecker and Lane[4] are also discussed. As the rationality of the proximity preservation is concerned, a splitting form which is weaker but rational is formulated. The reformulation makes the proximity preservation more like a type of the Pareto principle than an analogy of the continuity.

The author would like to express his gratitude to the referee for the advice to extend Theorem 1 to the infinite case and to relate proximity preservation with continuity. The original manuscript of this paper considered only the case of finite alternatives.

\section{Impossibility theorems}

Given two metric spaces $(X, \delta),(Y, d)$ and a function $F: X \rightarrow Y$, we say that $F$ is proximorphic (relative to the given metric $\delta$ and $d$ ), if

$$
\delta(x, y) \leq \delta(x, z) \text { implies } d(F(x), F(y)) \leq d(F(x), F(z)),
$$

for any $x, y, z$ in $X$. We write " $F$ satisfies PX relative to $\delta, d$ " for simplification.

For a metric space $W$ with metric $d$, sometimes denoted by $(W, d)$ for specification, let $W^{N}$ be the product space of $N$ copies of $W$ with the $L^{1}$ product metric $d^{N}$ defined by

$$
d^{N}\left(\left(a_{1}, \ldots, a_{N}\right),\left(b_{1}, \ldots, b_{N}\right)\right)=\sum_{i=1}^{N} d\left(a_{i}, b_{i}\right) .
$$

A function $F: W^{N} \rightarrow W$ is called diagonally surjective if $F$ maps the diagonal points of $W^{N}$ onto $W$, i.e., $F(D)=W$, where $D$ is the diagonal set $\left\{(a, a, \ldots, a) \in W^{N} ; a \in W\right\}$. The diagonal surjectivity is denoted by DS.

Theorem 1. Let $W$ be a set containing more than one element and let $F: W^{N} \rightarrow W, N>1$, be diagonally surjective. Then $F$ is not proximorphic, relative to any given metric $d$ of $W$ and its $L^{1}$-product metric $d^{N}$.

Proof. Clearly, there exist two diagonal points $(a, a, \ldots, a)$ and $(b, b, \ldots, b)$ in $W^{N}$ with distinct images

$$
F(a, a, \ldots, a) \neq F(b, b, \ldots, b) .
$$

Denote them by $x_{0}=(a, a, \ldots, a), x_{N}=(b, b, \ldots, b)$. Consider

$$
x_{1}=(a, a, \ldots, a, b) \in W^{N} .
$$

By the surjectivity DS, there exists a diagonal point $y=(c, c, \ldots, c, c) \in W^{N}$, with $F(y)=F\left(x_{1}\right)$. We have 


$$
\begin{aligned}
d^{N}\left(y, x_{1}\right) & =d^{N}((a, a, \ldots, a, b),(c, c, \ldots, c, c)) \\
& =(N-1) d(a, c)+d(c, b) \geq d(a, b)=d^{N}\left(x_{0}, x_{1}\right) .
\end{aligned}
$$

(The first inequality is by the triangular inequality and the last equality follows from $\mathrm{d}(\mathrm{a}, \mathrm{a})=0$.) Suppose $F$ is proximorphic, we have

$$
0=d\left(F(y), F\left(x_{1}\right)\right) \geq d\left(F\left(x_{0}\right), F\left(x_{1}\right)\right)
$$

and hence $F\left(x_{0}\right)=F\left(x_{1}\right)$. Let $x_{2}=(a, a, \ldots, a, b, b) \in W^{N}$. It is clear that $d^{N}\left(x_{1}, x_{2}\right)=d^{N}\left(x_{0}, x_{1}\right)$. Again by $F$ proximorphic,

$$
d\left(F\left(x_{1}\right), F\left(x_{2}\right)\right)=d\left(F\left(x_{0}\right), F\left(x_{1}\right)\right)=0,
$$

and we have $F\left(x_{2}\right)=F\left(x_{1}\right)=F\left(x_{0}\right)$. Do this process inductively, it is shown that $F\left(x_{N}\right)=F\left(x_{0}\right)$, leading to a contradiction. The proof is completed.

Example 2.1 We construct a nontrivial example for which $F: W^{2} \rightarrow W$ is proximorphic but not diagonally surjective. This will show that the assumption of diagonal surjectivity could not be dropped in Theorem 1. Let $W \equiv\left\{a, b, c_{1}, c_{2}, \ldots, c_{h}\right\}$ with $h \geq 9$ be a discrete set consisting of $h+2$ elements, and let the metric $d$ of $W$ be defined by

$$
\begin{aligned}
& d(a, b)=100, \quad d\left(a, c_{i}\right)=90, \quad d\left(b, c_{i}\right)=10, \\
& d\left(c_{i}, c_{j}\right)= \begin{cases}0 ; & i=j \\
1 ; & i \neq j,\end{cases}
\end{aligned}
$$

$\forall i, j \in\{1,2, \ldots, h\}$. Let $X_{1}, X_{2}, \ldots, X_{9}$ denote the nine sets in $W^{2}$ :

$$
\begin{aligned}
& \{(a, a)\},\{(b, b)\},\{(a, b)\},\{(b, a)\}, \\
& \left\{\left(c_{i}, a\right) ; i=1 \ldots, h\right\},\left\{\left(c_{i}, b\right) ; i=1, \ldots, h\right\} \\
& \left\{\left(a, c_{i}\right) ; i=1 \ldots, h\right\},\left\{\left(b, c_{i}\right) ; i=1, \ldots, h\right\} \\
& \left\{\left(c_{i}, c_{j}\right) ; i, j=1, \ldots, h\right\},
\end{aligned}
$$

respectively. Define $F: W^{2} \rightarrow W$ by $F(x)=c_{i}, \forall x \in X_{i}, i=1,2, \ldots, 9$. Evidently, $F$ is proximorphic, while not diagonally surjective.(For instance, the element $a$ is not contained in the image of $F$ ).

Baigent (1987) defined the notion of proximity preservation in the following weaker sense. Given a set $W$, a metric $d$ on $W$ and a function $F: W^{N} \rightarrow W, N>1$. We say that $F$ is weakly proximorphic relative to $d$, if

$$
d^{N}(x, y)<d^{N}(x, z) \text { implies } d(F(x), F(y)) \leq d(F(x), F(z)),
$$

for any $x, y, z$ in $W^{N}$. (Note that the inequality $(\leq)$ in the antecedent of (2.1) is here replaced by the strict inequality $(<)$.) Sometimes we write " $F$ satisfies WPX relative to $d$ " for simplication. Baigent defined that a social welfare function $F: W^{N} \rightarrow W$ preserves proximity when (2.3) is valid, i.e. when $F$ is weakly proximorphic relative to the given metric of $W$. The natural question is to determine whether Theorem 1 still holds for a weak proximorphism $F$.

For $N=2$, the answer is negative, since we may have an example of $F: W^{2} \rightarrow W$ which is diagonally surjective and weakly proximorphic as follows. 
Example 2.2 Let $W$ be a space in which all pairs of the elements are equally distanced with metric $d$, i.e. $d(a, b)=d\left(a^{\prime}, b^{\prime}\right)$ for any two pairs $a \neq b$ and $a^{\prime} \neq b^{\prime}$ in $W$. Let $F: W^{2} \rightarrow W$ be defined by the projection map to the second coordinate, i.e.

$$
F(a, b)=b, \quad \forall a, b \in W .
$$

Clearly $F$ is diagonally surjective. Also $F$ satisfies WPX relative to $d$. In fact, suppose the contrary, then there exist $x, y, z$ in $W^{2}$ such that

$$
d^{2}(x, y)<d^{2}(x, z) \text { and } d(F(x), F(y))>d(F(x), F(z)) .
$$

Since $W$ is equally distanced, the only possibility is that

$$
d(F(x), F(y)) \neq 0 \text {, but } d(F(x), F(z))=0,
$$

which yields that $F(x) \neq F(y)$ and $F(x)=F(z)$. Denote $x=(a, b)$, then $z=(c, b)$. Let $y=\left(a^{\prime}, b^{\prime}\right)$ then $b \neq b^{\prime}$. By $d^{2}(x, y)<d^{2}(x, z)$, we have

$$
d\left(a, a^{\prime}\right)+d\left(b, b^{\prime}\right)<d(c, a)
$$

which implies $c \neq a$. Since the elements of $W$ are equally distanced, $d(c, a)=d\left(b, b^{\prime}\right)$. We have

$$
d(c, a) \leq d\left(a, a^{\prime}\right)+d\left(b, b^{\prime}\right)<d(c, a),
$$

which is a contradiction. Hence $F$ satisfies WPX.

However, we will show for $N \geq 3$ that DS still precludes WPX, and for $N=2$ the only possibility is basically the case described in Example 2.2.

Definition 2.1. Given a metric space $W=(W, d)$ and $F: W^{N} \rightarrow W$, we call $F$ is simple if all the pairs of the elements of $W$ are equally distanced, and $F$ is a permuted projection, i.e. there is some $i$ such that $F\left(p_{1}, \ldots, p_{N}\right)=\pi\left(p_{i}\right)$, where $\pi$ is a permulation among elements of $W$.

Theorem 2. ${ }^{3}$ Let $W$ be a metric space containing more than one element and $F: W^{N} \rightarrow W, N>2$, be diagonally surjective, Then $F$ does not preserve proximity in the sense of Baigent (or equivalently, $F$ is not weakly proximorphic relative to the given metric $d$ and $L^{1}$-product metric $d^{N}$ ).

Proof. The proof is essentially the same with Theorem 1, by some modifications. Suppose $F$ is weakly proximorphic. We will show a contradiction. Clearly, there exist two distinct $a, b$ in $W$ such that $F(a, a, \ldots, a) \neq$ $F(b, b, \cdots, b)$. Given a natural number $l<N$. Consider

$$
x_{l}=(a, a, \ldots, a, b, b, \ldots, b) \in W^{N}
$$

\footnotetext{
3 The proof of Baigent's Theorem given in his paper (1987) seems to have a problem when $N$ is an odd number. Our proof of Theorem 2, when viewed as a generalization of Baigent's Theorem, automatically fixes the gap.
} 
with first $l$ coordinates taking $a$ and the rest taking $b$. By DS, there exists $y=(c, c, \ldots, c) \in W^{N}$ with $F(y)=F\left(x_{l}\right)$. Since

$$
\begin{aligned}
d^{N}\left(x_{l}, y\right) & =l d(a, c)+(N-l) d(b, c) \\
& >d(a, c)+d(b, c) \quad \text { as } 1 \leq l \leq N-1 \\
& \geq d(a, b)=d^{N}\left(x_{l}, x_{l+1}\right),
\end{aligned}
$$

(noting that $N>2$ is necessary to obtain the strict inequality in the last formula). We have

$$
0=d\left(F\left(x_{l}\right), F(y)\right) \geq d\left(F\left(x_{l}\right), F\left(x_{l+1}\right)\right),
$$

by WPX. Hence $F\left(x_{l}\right)=F\left(x_{l+1}\right)$. Similarly $F\left(x_{l-1}\right)=F\left(x_{l}\right)$. Therefore,

$$
F(a, a, \ldots, a)=F\left(x_{N}\right)=F\left(x_{N-1}\right)=\ldots=F\left(x_{0}\right)=F(b, b, \ldots, b),
$$

which contradicts $F(a, a, \ldots, a) \neq F(b, b, \ldots, b)$.

Theorem 3. Given $W, F$ as in Theorem 2, except now $N=2$. The only case in which $F$ preserves proximity in the sense of Baigent is when $F$ is simple.

Proof. See Appendix II.

\section{Continuity}

In this section, we deal with the maps on continuum metric spaces, and consider the relationship between the proximity preservation and continuity. In general, the continuity of a map means the preservation of infinitesimal proximity which is simply topological, while the proximity preservation is defined metrically. Indeed, proximity preservation concerns the orders of finiteness (e.g., $3<5$ ) under the map, while the continuity differentiates the order of infinitesimal smallness from orders of finiteness(e.g., $o(1)<1 / 9)$.

The two notions appear irrelevant to each other. This is seen by the observation that a rubber-bending which is a continuous map preserving the infinitesimal proximity may easily break the proximity, i.e. continuity does not imply proximity preservation. On the other hand, one may show that proximity preservation does not imply continuity, by constructing a scattering function which maps all of the elements in the domain into a totally discrete subset of the range such that the image points are distanced from each other in at least a constant range(see Defition 3.2). However, we prove that the scatterness example is the only case that a proximity preservation is not continuous.

Definition 3.1. A topological space $X$ is called a P-space if each component of $X$ is path-connected and isolated. ${ }^{4}$

\footnotetext{
${ }^{4}$ See for example [8] Kelley (1955). A component of $X$ is a maximal connected set of $X$. A path of $X$ means a continuous map from an interval into the space $X$. A set $Y$ in $X$ is path-connected, if $\forall x, y$ in $Y$, there is a path $\gamma:[a, b] \rightarrow Y$ such that $\gamma(a)=x, \gamma(b)=y$. A set $Y$ in $X$ is isolated if $Y$ is open and closed in $X$.
} 
Example 3.1. A discrete space is a P-space. An open set of an Eulidean space $\mathbb{R}^{n}$ or of a Hilbert space is a P-space.

Example 3.2. The Chichilnisky space is a P-space. Let $Q^{n}$ be a cube in $\mathbb{R}^{n}$. According to Chichilnisky (1982, [3]), a preference $v$ on $Q^{n}$ is defined by an unit continuous vector field on $Q^{n}$ except on a singular subset of $Q^{n}$ where $v=0$. Define the metric $d_{c}$ by

$$
d_{c}(v, w)=\max \left\{|v(x)-w(x)| ; x \in Q^{n}\right\}
$$

The totality $P_{c}$ of the preferences on $Q^{n}$, equipped with the metric $d_{c}$ which defines a topology of $P_{c}$, is called the Chichilnisky space. Given $v \in P_{c}$, the subset $K_{v}$ of $Q^{n}$ consisting of all the points on which $v$ is zero, is called the singular set of $v$, and $Q^{n}-K_{v}$ the regular set of $v$. Given $v, w \in P_{c}$. If $v$ and $w$ have different singular sets, then $d_{c}(v, w) \geq 1$. Two preferences $v$ and $w$ are in the same component of $P_{c}$, only when their singular sets are identical. But the converse is not true. For a counter example, let $n=2$, consider $Q^{2}=\left\{x=\left(x_{1}, x_{2}\right) \in \mathbb{R}^{2} ;\left|x_{i}\right| \leq 1\right\}$, and $v, w$ defined by

$$
v(x)=\left\{\begin{array}{l}
\frac{x}{|x|} ; \quad x \neq 0 \\
0 ; \quad x=0
\end{array} \quad w(x)=\left\{\begin{array}{l}
(1,0) ; \quad x \neq 0 \\
0 ; \quad x=0
\end{array}\right.\right.
$$

We have $d_{c}(v, w)=2$ and $K_{v}=K_{w}$, but $v$ and $w$ belong to different component. Clearly, two components of $P_{c}$ are distanced by at least 1 from each other. Hence each component $P_{c}$ is isolated. The path-connectedness of components of $P_{c}$ is evident.

Example 3.3. Every product space with $N$ copies of $\mathrm{P}$-spaces is also a P-space.

Theorem 4. Let $X$ and $Y$ be $P$-spaces with metrics $\delta$ and $d$ respectively. Let $Y$ have a countable (topological) base ${ }^{5}$. If a map $F: X \rightarrow Y$ is weakly proximorphic ${ }^{6}$ (i.e. satisfies $\left.W P X\right)$, relative to the given $\delta$ and $d$, then $F$ is continuous.

Proof. Given $\bar{x} \in X$, we will show that $F$ is continuous at $\bar{x}$. We say that $F$ is discrete at $\bar{x}$ if $\exists \varepsilon_{0}>0$ such that $\forall x^{\prime} \in X$ with $x^{\prime} \neq \bar{x}$, we have

$$
d\left(F\left(x^{\prime}\right), F(\bar{x})\right) \geq \varepsilon_{0} .
$$

The positive number $\varepsilon_{0}$ is called a scale of the discreteness of $F$. There occur two cases:

\footnotetext{
${ }^{5}$ A base of a topological space $\mathrm{X}$ is a family $\Phi$ of open sets which generates the topology of $X$, i.e. any open set in $X$ is a union of open sets of $\Phi$. For examples, an Euclidean space and even the product space of its countably many copies have a countable base.

${ }^{6}$ It means that $F$ satisfies: $\delta(x, y)>\delta(x, z) \Rightarrow d(F(x), F(y)) \geq d(F(x), F(z)), \forall x, y, z \in$ $X$. This is a generalization of (2.3) where $X=Y^{N}$. One may refer to the original definition of Baigent [2].
} 
Case 1. $F$ is not discrete at $\bar{x}$, i.e. $\forall \varepsilon>0, \exists x^{\prime} \in \mathbb{X}$ with $x^{\prime} \neq \bar{x}$, such that $d\left(F\left(x^{\prime}\right), F(\bar{x})\right)<\varepsilon$ :

Choose $\delta_{0}=\delta\left(x^{\prime}, \bar{x}\right)$. Then

$$
\delta(x, \bar{x})<\delta_{0} \Rightarrow \delta(x, \bar{x})<\delta\left(x^{\prime}, \bar{x}\right) .
$$

By WPX, we have $d(F(x), F(\bar{x})) \leq d\left(F\left(x^{\prime}\right), F(\bar{x})\right)<\varepsilon$. This proves $F$ continuous at $\bar{x}$ for Case 1 .

Case 2. $F$ is discrete at $\bar{x}$ :

Step 1. Let $X_{\alpha}$ denote the component of $X$ containing $\bar{x}$. We claim that $F$ is uniformly discrete on $X_{\alpha}$ with scale $\varepsilon_{0}$, i.e. $\forall x \in X_{\alpha}, F$ is discrete at $x$ and the scale $\varepsilon_{0}$ is independent of $x$. it suffices to consider only $x \neq \bar{x}$. Given $x^{\prime} \in X$ with $x^{\prime} \neq x$, if

$$
\delta\left(x, x^{\prime}\right)>\delta(x, \bar{x})
$$

then by WPX, $d\left(F(x), F\left(x^{\prime}\right)\right) \geq d(F(x), F(\bar{x})) \geq \varepsilon_{0}$. For the rest $x^{\prime} \in X$ with $x^{\prime} \neq x$, and

$$
\delta\left(x, x^{\prime}\right) \leq \delta(x, \bar{x})
$$

Connect $x$ to $\bar{x}$ by a path $\gamma$ and select points $x_{1}, x_{2}, \cdots, x_{k}$ on $\gamma$ such that

$$
\delta\left(x, x^{\prime}\right)>\delta\left(x, x_{1}\right)>\delta\left(x_{1}, x_{2}\right)>\ldots>\delta\left(x_{k-1}, x_{k}\right)>\delta\left(x_{k}, \bar{x}\right) .
$$

By successively applying WPX, we have

$$
d\left(F(x), F\left(x^{\prime}\right)\right) \geq \cdots \geq d\left(F\left(x_{k}, F(\bar{x})\right)\right) \geq \varepsilon_{0} .
$$

Hence $F$ is discrete at any point $x$ in $X_{\alpha}$. Furthermore, the scale $\varepsilon_{0}$ is independent of $x$. The claim is proved.

Step 2. If $X_{\alpha}$ is a singleton set (i.e. containing exactly one element $\bar{x}$ ), then $X_{\alpha}$ is isolated by the definition of P-space. It is automatically true that $F$ is continuous at $\bar{x}$. On the other hand, if $X_{\alpha}$ is not a singleton set, then $X_{\alpha}$ is an uncountable set, since $X_{\alpha}$ is path-connected and the image of a nontrivial path is an uncountable set. By Step 1, we have shown that for any $x \in X_{\alpha}$, there is no point of $X$ other than $x$ itself is mapped into the $\varepsilon_{0}$-neighborhood of $F(x)$. Hence there $\varepsilon_{0}$-neighborhoods of $F(x), x \in X_{\alpha}$, are disjoint from each other. It yields that $Y$ has uncountable disjoint nonempty open balls of radius $\varepsilon_{0}$, contradicting to the hypothesis that $Y$ has countable base. Therefore Case 2 is impossible.

Thus we have shown that $F$ is continuous at $\bar{x}$. The proof is completed.

Corollary 1. Let $U^{m}$ be an open set in $\mathbb{R}^{m}$ and $F: U^{m} \rightarrow \mathbb{R}^{k}$ is weakly proximorphic (relative to a given metric which is topologically equivalent to the Euclidean metric). Then $F$ is continuous. 
Proof. Since $U^{m}$ is a P-space and $\mathbb{R}^{k}$ has a countable base, Theorem 4 is applicable to this case.

Definition 3.2. Given $Z \subset X$ and $F: X \rightarrow Y, X$ and $Y$ being metric spaces, we call $F$ scattering on $Z$, if $\forall x \in Z$ and $\forall x^{\prime} \in X$ with $x \neq x^{\prime}$, we have

$$
d\left(F(x), F\left(x^{\prime}\right)\right)>\sigma,
$$

where $\sigma$ is called the scale of the scattering.

Let $Z \subset X$ and $F: X \rightarrow Y$. Clearly, $F$ is scattering on $Z$ with scale $\sigma$, iff $F$ is uniformly discrete on $Z$ with the same scale $\sigma$. The proof of Theorem 4 yields the following corollary.

Corollary 2. Let $F: X \rightarrow Y, X$ and $Y$ being $P$-spaces. If $F$ is weakly proximorphic, then given any component $X_{\alpha}$ of $X, F$ is either continuous on $X_{\alpha}$ or scattering on $X_{\alpha}$.

Theorem 5. Let $F: P_{c}^{N} \rightarrow P_{c}, N>0$, be weakly proximorphic, where $P_{c}$ is the Chichilnisky space. Then for a given component $X_{\alpha}$ of $P_{c}^{N}, F$ is either continuous on $X_{\alpha}$, or scattering on $X_{\alpha}$ with scale $\sigma=1$. In particular, given a social welfare function $F: P_{c}^{N} \rightarrow P_{c}$ where $F$ is onto, we have

$F$ is weakly proximorphic $\Rightarrow F$ is continuous

Proof. Step 1. Denote $\tilde{0}=(0, \cdots, 0) \in P_{c}^{N}$. We may consider without loss of generality that $X_{\alpha} \neq\{\tilde{0}\}$, since $\tilde{0}$ is an isolated point in $P_{c}^{N}$ and therefore $F$ is automatically continuous at $\tilde{0}$. We note that

$$
d_{c}^{N}(\tilde{p}, \tilde{q}) \leq 2 N, \forall \tilde{p}, \tilde{q} \in X_{\alpha}
$$

and $2 N$ is the diameter of $P_{c}^{N}$ (i.e. the maximal distance among elements in $P_{c}^{N}$ ). By Corollary 2, $F$ is either continuous or scattering on $X_{\alpha}$. If $F$ is not continuous on $X_{\alpha}$, we claim that the scattering scale $\sigma$ is 1 . Let $\tilde{p} \in X_{\alpha}$. Denote the component of $P_{c}$ containing $F(\tilde{p})$ by $Y_{a}$. Consider the set

$$
B \equiv\left\{\tilde{r} \in X_{\alpha} ; F(\tilde{r}) \notin Y_{a}\right\} .
$$

Since $F$ is scattering on $X_{\alpha}$ and $Y_{a}$ has only a countable base, $X_{\alpha}-B$ contains at most countable elements. But $X_{\alpha}$ is an uncountable set. Hence $B$ is dense in $X_{\alpha}$ and there exist elements in $B$ which are arbitrarily close to $\tilde{p}$ in $P_{c}^{N}$. Given $\tilde{q} \in X$, we may select $\tilde{r} \in B$ with

$$
d_{c}^{N}(\tilde{p}, \tilde{q})>d_{c}^{N}(\tilde{p}, \tilde{r}) .
$$

By $F$ weakly proximorphic, we have $d_{c}(F(\tilde{p}), F(\tilde{q})) \geq d_{c}(F(\tilde{p}), F(\tilde{r}))$. But $F(\tilde{r})$ belong to a component different from $Y_{a}$. Any two components of $P_{c}$ are distanced from each other by 1 . So $d_{c}(F(\tilde{p}), F(\tilde{q})) \geq 1$ as claimed.

Step 2. It is not difficult to see that $P_{c}$ has a base with cardinality $\aleph_{2}$. In particular, $P_{c}$ has no countable base. Hence (3.3) can not be proved directly by applying Theorem 4 . However, when $F$ is onto, $F$ is clearly not scattering 
on a given component of $P_{c}^{N}$. By Step 1, this yields that $F$ is continuous on each component of $P_{c}^{N}$ and the proof of Theorem 5 is completed.

\section{Rigidity}

It is interesting to learn more about the proximity preservation. We establish a series of theorems in this section. The object is two folded. The theorems depict the geometry of the proximity preservation on general metric spaces which would clarify more closely the role of proximity preservation in social choice theory. Also, the theorems show that a weak proximorphism is almost a rigid motion (only up to a dilation).

Definition 4.1. A path in a metric space $X$ is a continuous map $\gamma:[a, b] \rightarrow X$, where $[a, b]$ is an interval in $\mathbb{R}^{1}$. A subpath $\gamma_{1}$ of $\gamma$ is the restriction of $\gamma$ to $a$ subinterval $\left[a_{1}, b_{1}\right]$ of $[a, b]$. A path $\gamma$ of $X$ is called minimizing if for any subpath $\gamma_{1}:\left[a_{1}, b_{1}\right] \rightarrow X$, and for any point $z$ on $\gamma_{1}$ (i.e. $z=\gamma_{1}(c), c \in\left[a_{1}, b_{1}\right]$ ), we have

$$
d_{X}\left(x_{1}, z\right)+d_{X}\left(z, y_{1}\right)=d_{X}\left(x_{1}, y_{1}\right) \text {, }
$$

where $x_{1}=\gamma_{1}\left(a_{1}\right), y_{1}=\gamma_{1}\left(b_{1}\right)$. A pair $\{x, y\}$ of points in $X$ is called path-minimizing, if there exists a minimizing path $\gamma$ connecting $x$ to $y$. A metric space $X$ is called path-minimizing if all pairs of points in $X$ is path-minimizing.

A convex domain in $\mathbb{R}^{n}$, a convex set in a Hilbert space, a connected complete surface, or a complete Riemannian manifold, are obvious examples of path-minimizing metric spaces. However, the circle $S^{1}$ with metric $d$ defined by the restriction of Euclidean metric in $\mathbb{R}^{2}$ is not path-minimizing. It is not difficult to prove the following theorems. The details of the proofs are sketched in Appendix III.

ET (Equivalence Theorem). Given an onto map $F: X \rightarrow Y$, where $X$ and $Y$ are path-minimizing metric spaces, we have the equivalence that $F$ is weakly proximorphic if and only $F$ is proximorphic.

PMP(Path-minimizing Preservation). Let $F: X \rightarrow Y$ be given as in ET. If $F$ is weakly proximorphic, then $F(\gamma)$ is a minimizing path in $Y$ for any minimizing path $\gamma$ in $X$.

We remark that if $F$ is not assumed to be onto, the path-minimizing preservation may not be true. This is seen by considering $F: \mathbb{R}^{1} \rightarrow \mathbb{R}^{2}$, where $F(x)=e^{2 \pi i x}, i=\sqrt{-1}$.

Definition 4.2. A map $F: X \rightarrow Y, X$ and $Y$ being metric spaces, is called similar if there exists a constant $c \geq 0$ such that

$$
d_{Y}(F(x), F(y))=c \cdot d_{X}(x, y),
$$

$\forall x, y$ in $X$. We call the constant $c$ the dilation coefficient.

If $c \neq 0$ and $F$ is onto, then the condition that $F$ is similar means that $F$ is isometric (i.e. $F$ is a rigid motion), up to a dilation of the metric space $Y$ (or $X$ ). Therefore the similarity is a very strict condition upon $F$. 
ST (Similarity Theorem). Let $F: X \rightarrow Y$ be given as in ET, and let $F$ be weakly proximorphic. Then $F$ is similar.

$T T$ (Triviality Theorem). Let $F: U \rightarrow \mathbb{R}^{m}, U$ being a domain in $\mathbb{R}^{n}, n>m$. Then $F$ never preserves proximity (i.e. $F$ can never be weakly proximorphic), unless $F$ maps the whole domain $U$ into a single point in $\mathbb{R}^{m}$.

Proof. By ST, $F$ is similar. However, two Euclidean spaces of different dimensions are not similar, unless the similarity map is trivial (i.e. $\mathrm{c}=0$ ).

As the Chichilnisky space $P_{c}$ and its product $P_{c}^{N}$ are not path-minimizing, we have to extend the study so that it deals with social welfare functions on $P_{c}$.

Definition 4.3. A metric space $X$ is locally-minimizing if for any $x \in X$, there exists a neighborhood $U$ of $x$ in $X$ which is path-minimizing.

Notice that $P_{c}$ is not even locally minimizing. In fact, consider $v$ and $w$ in $(n-1)$-sphere $S^{n-1}$, regarded as two constant unit vector fields on $Q^{n} \subset \mathbb{R}^{n}$. Clearly, for any $u \in S^{n-1}$,

$$
|v-w|<|v-u|+|u-w|,
$$

if they are all distinct. Therefore, $P_{c}$ is not locally path-minimizing. But if we replace the Chichilnisky metric $d_{c}$ by the angular metric $d_{\theta}$ which is defined by

$$
d_{\theta}(p, q)=\max _{x \in Q^{n}} \cos ^{-1}\langle p(x), q(x)\rangle,
$$

$\forall p, q \in P_{c}$, then $P_{c}$, as well as $P_{c}^{N}$, are locally path-minimizing, although they are still not path-minimizing. The notation $\langle p(x), q(x)\rangle$ in (4.2) denotes the Euclidean inner product, and $\cos ^{-1}$ takes the principal value in the range $[0, \pi]$.

Example 4.1. Consider $v, w$ in $P_{c}$ as follows. Let the alternative set be the square $Q^{2}=\{x=a+b i \in \mathbb{C} ; 0 \leq a \leq 1,0 \leq b \leq 1\} \subset \mathbb{R}^{2}$. Define

$$
v(x)=e^{i \theta(x)}, \theta(x)=\left(\frac{3 \pi}{2}\right) \sin \pi a, \quad \text { and } \quad w(x)=1 \in \mathbb{C},
$$

for any $x=a+b i \in Q^{2}$. Then $\langle v(x), w(x)\rangle=\cos \theta(x)$ and by (4.2),

$$
d_{\theta}(v, w)=\max _{x \in Q^{2}} \cos ^{-1}\langle v(x), w(x)\rangle=\max _{x \in Q^{2}} \tilde{\theta}(x),
$$

where $\tilde{\theta}(x)$ means the principal value of the arccos at $\cos \theta(x)$, such that $0 \leq \tilde{\theta}(x) \leq \pi$. Clearly, $d_{\theta}(v, w)=\pi$, since the maximal value of $\tilde{\theta}(x)$ is attained at $a_{0}=\frac{1}{\pi} \sin ^{-1} \frac{2}{3}$. Consider a path $\gamma(t)=e^{i t \theta}$, for $0 \leq t \leq 1$. Then $\gamma(t)$ is a path connecting $w$ to $v$. It is not minimizing, since if we take $t=\frac{2}{3}$, for example, and let $u=\gamma\left(\frac{2}{3}\right)$, then

$$
d(v, u)+d(u, w)=\frac{\pi}{2}+\pi>d(v, w) .
$$


This similar situation happens actually for any path connecting $w$ to $v$. Therefore the component of $P_{c}$, even relative to $d_{\theta}$, are not path-minimizing in general.

However, the angular metric $d_{\theta}$ determines the same topology for $P_{c}$ as that given by the Chichilnisky metric $d_{c}$. Also, a social welfare function $F: P_{c}^{N} \rightarrow P_{c}$ is a (weak) proximorphism relative to $d_{\theta}$ if and only if $F$ is a (weak) proximorphism relative to $d_{c}$. Therefore the replacement of $d_{c}$ by $d_{\theta}$ does not change the essence of $P_{c}$, in our consideration.

We conclude this paper by establishing the following theorem. The proof is also given in Appendix III.

LST (Local Similarity Theorem). Let $F: P_{c}^{N} \rightarrow P_{c}$, be a social welfare function in the Chichilnisky framework. If $F$ has no taboo(i.e. $F$ is onto) and it is weakly proximorphic relative to $d_{c}$ (or $d_{\theta}$ ), then $F$ is a local similar map relative to the angular metric $d_{\theta}$ with the dilation coefficient $c=\frac{1}{N}$.

\section{Concluding remarks}

It is nice that Baigent called my attention to the recent interesting works by Grafe and Grafe [5] and by Eckert and Lane [4], during the preparation of the final version of this paper. My argument of criticism about the proximity preservation is not applicable to their formulation. In [4], the ordinal proximity preservation is considered, where the distance is not even metrical. Ecker and Lane obtained a strong impossibility theorem that the ordinal proximity preservation which satisfies anonymity is imposed. Grafe and Grafe showed in [5] that the distance preservation, the unanimity and anonymity are inconsistant, where the distance on the space of profiles is defined by a general partially congruent metric which is not necessarily $d^{N}$ as in (2.3) introduced by Baigent. Both [4] and [5] assume anonymity, which is not taken here in this paper. As impossibility theorems, those in [4], [5] and Theorem 2 of this paper are the generalization of Baigent's theorem, on behalf of Baigent's original work. However, as long as the rationality of the proximity preservation is concerned, I have a feeling that all the known definitions in whatever sense (of [4], [5], or of Baigent's) are over-demanding. The geometric reasoning is roughly sketched as follows. Given a social welfare function $F:\left(\Omega^{I}, \delta\right) \rightarrow(Y, d)$, which satisfies the proximity preservation in any of the above sense (abbrev. PP in the following). Consider the preimage $F^{-1}(y)$ for a given $y \in Y$. Let $A, B \in F^{-1}(y)$ be distinct. Then any $C \in \Omega^{I}$, which is sufficiently close to $A$, e.g. $\delta(A, C)<\delta(A, B)$, lies in $F^{-1}(y)$ by PP. In this way, $F^{-1}(y)$ would enlarge itself until it covers the whole $\Omega^{I}$, if some additional mild condition (e.g. the anonymity or the diagonal surjectivity) is satisfied to support the existence of the assumed $C$. This plausible reasoning although not rigorous however reveals the essence of the proximity preservation. It may apply even for non-congruent metrics such as $d_{3}=\max \left\{\delta_{i} ; i \in I\right\}$ in [5]. In the formulation of the proximity preservation, it seems to me that the integrated distance on the profile space should be reconsidered. Perhaps it is more 
reasonable to define the proximity preservation by comparing the component distance of individual preferences, i.e. by setting

$$
d\left(A_{i}, B_{i}\right)>d\left(A_{i}, C_{i}\right), \forall i \Rightarrow d(F(A), F(B)) \geq d(F(A), F(C)),
$$

instead of letting $\delta(A, B)>\delta(A, C)$ in the hypothesis. I think that the change is crucial in social choice theory.

A good impossibility theorem involving PP should have the formulation of PP to match at least two criterions:

1. Independence of the chosen metrics $\delta$ and $d$ - this should be required, since there is no distance a priori given.

2. Existence of abundant possible examples of a SWF satisfying PP - thus PP can be called a rational principle.

Criterion 1 is important, because if the impossibility theorem is valid only when the distance is chosen in a specific way, then it hardly concludes that a rational social choice is impossible, since comparison of the distances in PP become artificial. The results in [4] and [5] fit Criterion 1 satisfactorily. Especially, Eckert and Lane formulated $\delta$ and $d$ in a large class of binary relations, which are non-metrical and not even weak orders, and therefore make the impossibility theorem greatly successful at this point. However, in Baigent's original formulation of PP, the integrated distance $\delta$ which is the $L_{1}$ norm $d^{N}$ is perhaps too specific, while $d$, used as the individual and social distance, ranging over all possible distances, has no restriction. Criterion 2 says that a rational principle should not be too strict. It is one of the main task of this paper to show that PP in the original formulation (2.3) seems not to match this criterion, e.g. we have shown that a social welfare function satisfying PP in the sense of Baigent is almost rigid (see ST and LST in §4). The above reasoning stated in the first paragraph of this section also suggests that PP defined in the sense of [4] and [5] is not easy to be satisfied unless $F$ is trivial (e.g. imposed). This happens especially when $Y$ is absolutely "smaller" than $\Omega^{I}$, which is the canonical case considered in social choice theory, e.g., $Y=\Omega$, smaller than $\Omega^{I}$ in their dimensions. We have constructed in Example 2.1 a non-trivial social welfare function, but it has to be strangely peculiar which I wonder nevertheless is a typical case. One would convince himself that SWF satisfying PP is "rare", if he has ever tried to construct a non-trivial example.

On the other hand, the proximity preservation PP is not an analogy of continuity. This has been shown in the beginning paragraphs of 3 , by that the levels of the orders which they compare are different. PP is originally intended to capture the idea that "small changes in individual preferences should not lead to large changes in the social preferences" (see [2], [4]). A symmetric statement that large changes in individual preferences should not lead to small changes in the social preferences immediately follows by the formulation of PP. However, the symmetric statement is not true for continuous map in general. Look at a nice continuous function $F: \mathbb{R}^{N} \rightarrow \mathbb{R}$ with $N>1$. Let $A, B$ belong to 
a level set $F^{-1}(y), y \in \mathbb{R}$. In general, the change from $A$ to $B$ can be very large, but the change from $F(A)$ to $F(B)$ is as small as zero. More precisely, choose $C \notin F^{-1}(y)$, so close to $A$ that $d^{N}(A, C)<d^{N}(A, B)$. Such point $C$ exists if $F$ is not trivial. Then by PP, $d(F(A), F(C)) \leq d(F(A), F(B))=0$ and $F(A)=F(C)$. Therefore $C \in F^{-1}(y)$, which is a contradiction.

In summary, the reason of re-formulating PP into the splitting form (5.1) is the following.

1. Specific integrated distance $\delta$ on profiles is not a priori given.

2. PP in the original formulation is too strict and there are rare nontrivial social welfare functions satisfying PP.

3. PP and continuity are not analogous.

4. PP in the splitting form (5.1) free of the integrated distance would avoid the weak points (1) and (2) and still reasonable. The splitting form is more related to a type of Pareto principle or to a weaker form of unanimity than to continuity: i.e. given three profiles $A, B, C$, if for every individual $i$, $\left(A_{i}, C_{i}\right)$ is closer to each other than $\left(A_{i}, B_{i}\right)$, so it happens for the corresponding social preferences.

The splitting re-formulation of the proximity preservation is much weaker but clearly rational.

\section{Appendix}

\section{Review of definitions:}

Given $X$ a set which denotes the set of alternatives. By a preference $p$ on $X$ we mean a binary relation $\succ$ on $X$, such that

(i)(Completeness) $\forall x, y \in X$, either $x \gtrsim y$ or $y \succ x$ or both,

(ii)(Transitivity) $\forall x, y, z \in X, x \succ y$ and $y \succ z \Rightarrow x \succ z$.

The totality $F$ of all the preferences on $X$ is called the preference space. Let $V=\left\{v_{1}, v_{2}, \ldots, v_{N}\right\}$ be a finite set of individuals who act as voters in a social aggregation process. By a profile

$$
\tilde{p}=\left(p_{1}, p_{2}, \ldots, p_{N}\right)
$$

we mean an $\mathrm{N}$-tuple of individual preferences $p_{i}$, where for each $i, p_{i} \in P$ is the preference of the voter $v_{i}$. The totality of profiles is denoted by $P^{N}$. A social welfare function $F$ is a function

$$
F: P^{N} \rightarrow P \text {. }
$$

We review the following definitions.

(1) $F$ is called unanimous, if $F(p, p, \cdots, p)=p, \forall p \in P$.

(2) $F$ is called anonymous, if $F\left(p_{1}, p_{2}, \ldots, p_{N}\right)=F\left(p_{\sigma_{1}}, p_{\sigma_{2}}, \ldots, p_{\sigma_{N}}\right)$, $\forall\left(p_{1}, p_{2}, \ldots, p_{N}\right) \in P^{N}$ and $\forall$ permutation $\sigma$ of the indices $1,2, \ldots, N$.

(3) $F$ preserves proximity (relative to a given metric on $P$ ) if 


$$
d^{N}(\tilde{p}, \tilde{q})<d^{N}(\tilde{p}, \tilde{r}) \Rightarrow d^{N}(F(\tilde{p}), F(\tilde{q})) \leq d^{N}(F(\tilde{p}), F(\tilde{r}))
$$

$\forall \tilde{p}, \tilde{q}_{i}, \tilde{r} \in P^{N}$ where $d^{N}$ is defined by

$$
d^{N}(\tilde{p}, \tilde{q})=\sum_{i=1}^{N} d\left(p_{i}, q_{i}\right) .
$$

(If $P$ is a topological space, the metric $d$ is required to respect the topology of $P)$. This definition is introduced by Baigent (1987). In this paper, a social welfare function $F$ is also called weakly proximorphic, if it preserves proximity.

\section{Proof of Theorem 3 in Sect. 1:}

Suppose $F$ is weakly proximorphic, relative to a metric $d$ of $W$. We show that $F$ is simple, in the following steps:

Step 1. Given any two distinct elements $a, b$ in $W$, it is claimed that

$$
F(a, b)=F(a, a) \text { or } F(b, b) .
$$

By DS, there exists $c \in W$ such that $F(a, b)=F(c, c)$. If the claim (II.1) is not true, then $c \neq a$ and $c \neq b$

$$
\begin{aligned}
d^{2}((a, b),(c, c)) & =d(a, c)+d(b, c) \\
& >d(b, c)=d^{2}((a, b),(a, c)) .
\end{aligned}
$$

By WPX, $\quad 0=d(F(a, b), F(c, c)) \geq d(F(a, b), F(a, c))$. Hence $F(a, b)=$ $F(a, c)$. Similarly, $F(a, b)=F(c, b)$. However, we have

$$
\begin{aligned}
d^{2}((a, c),(c, b)) & =d(a, c)+d(c, b)>d(c, b) \\
& =d^{2}((c, b),(b, b)) .
\end{aligned}
$$

Again by WPX, we have $F(c, b)=F(b, b)$. Hence $F(a, b)=F(c, b)=F(b, b)$, a contradiction.

Step 2. Given $a \neq b$ in $W$, if $F(a, b)=F(a, a)$, we claim that $F(b, a)=F(b, b)$. It is seen by Step 1 that $F(b, a)=F(a, a)$ or $F(b, b)$. Suppose that $F(b, a)=F(a, a)$. By applying WPX as the previous standard way on the two pairs $\{(b, a),(a, b)\}$ and $\{(b, a),(b, b)\}$, we have $F(b, a)=F(b, b)$ as claimed.

Step 3. Given $a \neq b$ in $W$, if all the four points $(a, a),(a, b),(b, a)$ and $(b, b)$ are mapped by $F$ to a same point $\xi$ in $W$, we say that the lattice $\mathscr{L}_{a, b}$ collapses to $\xi$. Now we claim that there is no lattice collapsing to a point. But it suffices to show that if a lattice $\mathscr{L}_{a, b}$ collapses to $\xi \in W$, then all the lattices collapse to $\xi$ and therefore $F\left(W^{N}\right)=\{\xi\}$, contradicting to the diagonal surjectivity 
and $W \neq$ singleton set. In fact, given $c, d \in W, c \neq d$, we will show first that the lattice $\mathscr{L}_{a, c}$ collapses to $\xi$. By Step 1 and 2, one of $(a, c)$ and $(c, a)$ should be mapped to $F(a, a)=\xi$. Let $F(a, c)=F(a, a)$. Applying WPX on the two pairs $\{(a, c),(b, a)\}$ and $\{(a, c),(c, c)\}$, we have $F(a, c)=F(c, c)$. Therefore $\xi=F(a, a)=F(a, c)=F(c, c)$ and clearly $\mathscr{L}_{a, c}$ collapses to $\xi$. Similar argument applies for the case that $F(c, a)=F(a, a)$. By transitivity, $\mathscr{L}_{c, d}$ also collapses to $\xi$.

Step 4. By Step 3, there is no lattice $\mathscr{L}_{a, b}$ collapsing. Hence $\forall a, b \in W$ with $a \neq b$, we have $F(a, a) \neq F(b, b)$. This implies $F$ is diagonally injective (denoted by DI). We now claim that all elements in $W$ are equally distanced. Suppose the contrary, there exist $a, b, c \in W$ such that

$$
d(a, b)>d(a, c) \text { and } d(a, b) \geq d(b, c),
$$

by choosing $a, b$ with longest distance among the three. By Step 1, we may let $F(a, b)=F(a, a)$. Applying WPX on the two pairs $\{(a, b),(a, a)\}$ and $\{(a, b),(c, b)\}$, we see that $F(c, b)=F(a, b)=F(a, a)$. Applying again WPX on the two pairs $\{(c, b),(a, a)\}$ and $\{(c, b),(b, b)\}$, we obtain

$$
F(b, b)=F(c, b)=F(a, a),
$$

contradicting to the diagonal injectivity.

Step 5. It remains to show that $F$ is a permuted projection. Let $a, b \in W, a \neq b$. By Step 1, we may assume without loss of generality that $F(a, b)=F(a, a)$.

We claim:

(i) $F(a, x)=F(a, a), \forall x \in W$,

(ii) $F(x, y)=F(x, x), \forall x, y \in W$.

Suppose (i) is false. By Step 1, $F(a, x)=F(x, x)$, By Step 2, $F(x, a)=F(a, a)$. Applying WPX on the two pairs $\{(a, b),(x, a)\}$ and $\{(a, b),(b, b)\}$, we have $F(b, b)=F(a, b)$, which means $\mathscr{L}_{a, b}$ collapsing to $F(a, a)$, contradicting to the fact stated in Step 3. Thus (i) is proved. To claim (ii), we see from (i) and Step 2 that $F(x, a)=F(x, x), \forall x \in W$. But this yields $F(x, y)=F(x, x), \forall x, y \in W$, by applying (i) again. This proves (ii). The proof of Theorem 3 is completed.

\section{Proofs of the theorems in Sect. 4:}

We sketch the proofs of the theorems as follows. For the equivalence theorem ET, it needs only to apply the continuity theorem (Theorem 4) and use a standard argument of the $\varepsilon-\delta$ calculus. To show path-minimizing preservation PMP, we consider a minimizing path $\gamma_{0}$ in $X$. Suppose $F\left(\gamma_{0}\right)$ is not minimizing. Then there is a subpath $\gamma^{\prime}=F(\gamma)$ of $F\left(\gamma_{0}\right)$ with end points $x^{\prime}$ and $y^{\prime}$ and we can find $z^{\prime}$ on $\gamma^{\prime}$ such that

$$
d_{Y}\left(x^{\prime}, z^{\prime}\right)+d_{Y}\left(z^{\prime}, y^{\prime}\right)>d_{Y}\left(x^{\prime}, y^{\prime}\right)
$$


where $z^{\prime}=F(z), x^{\prime}=F(x), y^{\prime}=F(y), \gamma$ is a subpath of $\gamma_{0}$ connecting $x$ to $y$ in $X$, and $z$ is on $\gamma$. We have

$$
d_{X}(x, z)+d_{X}(z, y)=d_{X}(x, y) .
$$

Since $Y$ is also path-minimizing, there is a minimizing path $\alpha$ connecting $x^{\prime}$ to $y^{\prime}$ in $Y$. Choose a point $w^{\prime}$ on $\alpha$, such that the following two ratios are equal:

$$
d_{Y}\left(x^{\prime}, w^{\prime}\right): d_{Y}\left(w^{\prime}, y^{\prime}\right)=d_{Y}\left(x^{\prime}, z^{\prime}\right): d_{Y}\left(z^{\prime}, y^{\prime}\right) .
$$

By $F$ onto, there exists $w \in X$ such that $F(w)=w^{\prime}$. Clearly,

$$
d_{Y}\left(x^{\prime}, w^{\prime}\right)+d_{Y}\left(w^{\prime}, y^{\prime}\right)=d_{Y}\left(x^{\prime}, y^{\prime}\right)<d_{Y}\left(x^{\prime}, z^{\prime}\right)+d_{Y}\left(z^{\prime}, y^{\prime}\right) .
$$

By (III.2), $d_{Y}\left(x^{\prime}, w^{\prime}\right)<d_{Y}\left(x^{\prime}, z^{\prime}\right)$ and $d_{Y}\left(w^{\prime}, y^{\prime}\right)<d_{Y}\left(z^{\prime}, y^{\prime}\right)$. Then we have

$$
d_{X}(x, w)<d_{X}(x, z) \text { and } d_{X}(w, y)<d_{X}(z, y),
$$

since $F$ is proximorphic by ET. Hence

$$
d_{X}(x, w)+d_{X}(w, y)<d_{X}(x, z)+d_{X}(z, y)=d_{X}(x, y),
$$

contradicting to the triangular inequality of the metric $d_{X}$. This completes the proof of PMP. To claim the similarity theorem ST, we have to show that for a given pair $\{x, y\}$ in $X$, if $d_{Y}(F(x), F(y))=c \cdot d_{X}(x, y)$ with $c \geq 0$, then

$$
d_{Y}(F(z), F(w))=c \cdot d_{X}(z, w),
$$

for all $z, w$ in $X$. Consider minimizing paths $\gamma, \alpha$, and $\beta$ which respectively connect $x$ to $y, x$ to $z$ and $z$ to $w$. We first claim that

$$
d_{Y}(F(x), F(u))=c \cdot d_{X}(x, u), \forall u \text { on } \gamma .
$$

Assume that the numbers $d_{X}(x, u)$ and $d_{X}(x, y)$ are commensurable, then there exist $u_{1}$ on $\gamma$ such that

$$
d_{X}(x, u)=m d_{X}\left(x, u_{1}\right) \text { and } d_{X}(x, y)=n d_{X}\left(x, u_{1}\right),
$$

with two integers $m$ and $n$. In fact, we subdivide $\gamma$ with subdivision points $u_{1}, u_{2}, \ldots, u_{m}, \ldots, u_{n}$, such that

$$
d_{X}\left(x, u_{1}\right)=d_{X}\left(u_{1}, u_{2}\right)=\ldots=d_{X}\left(u_{n-1}, u_{n}\right)
$$

where $u=u_{m}, y=u_{n}$. By ET, $F$ weakly proximorphic implies $F$ is proximorphic. Since $F(\gamma)$ a minimizing path, we can show that

$$
d_{Y}(F(x), F(y))=n \cdot d_{Y}\left(F(x), F\left(u_{1}\right)\right) .
$$

and

$$
\begin{aligned}
d_{Y}(F(x), F(u)) & =m \cdot d_{Y}\left(F(x), F\left(u_{1}\right)\right)=\frac{m}{n} \cdot d_{Y}(F(x), F(y)) \\
& =\frac{m}{n}\left(c \cdot d_{X}(x, y)\right)=c \cdot d_{X}(x, u),
\end{aligned}
$$

where the last equality follows from (III.5). Hence (III.4) is proved, by applying the continuity of $F$ to cover the general $u$. Next, we claim (III.3). 
Assume $d_{X}(x, y), d_{X}(x, z)$ and $d_{X}(z, w)$ are commensurable. Then there exist $u_{1}$ on $\gamma, v_{1}$ on $\alpha$ and $w_{1}$ on $\beta$ such that

$$
d_{X}(x, y)=k \cdot d_{X}\left(x, u_{1}\right), d_{X}(x, z)=l \cdot d_{X}\left(z, v_{1}\right), d_{X}(z, w)=p \cdot d_{X}\left(z, w_{1}\right),
$$

where $k, l, p$ are integers. By the same reasoning as above, we can show (III.3), which would complete the proof of ST.

Finally, the local similarity theorem LST is a direct application of the similarity theorem. The dilation coefficient $c$ is constant on any given component of $P_{c}^{N}$. It is not difficult to show that $c=\frac{1}{N}$. The argument is based on the onto assumption of $F$.

\section{References}

[1] Arrow KJ (1951) Social choice and individual values. Wiley, New York

[2] Baigent N (1987) Preference proximity and anonymous social choice. Q J Econ 102: $161-169$

[3] Chichilnisky G (1982) Social aggregation rules and continuity. Q J Econ 97(2): 337-352

[4] Grafe F, Grafe J (2001) Social welfare functions which preserve distances. Soc Choice Welfare 18: 59-64

[5] Eckert D, Lane B (2002) Anonymity, ordinal preference proximity and imposed social choices. Soc Choice Welfare 19: 681-684

[6] Huang WH (1997) Singularities in social choice theory. Technical Report, Mathematical Department, National Taiwan University

[7] Huang WH (1998) Singularities and continuity of social choice on continuum spaces (forthcoming)

[8] Kelley J (1955) General topology. Van Nostrand, New Jersey

[9] Sen A (1986) Social choice theory. In: Arrow KJ, Intriligator M (eds) Handbook of mathematical economics, V.III. North-Holland, Amsterdam 